\title{
Economic assessment in minimally invasive thoracic oncological surgery-USA experience
}

\author{
Jonathan Cools-Lartigue ${ }^{1}$, Bernard J. Park ${ }^{2}$ \\ ${ }^{1}$ Department of Thoracic Surgery, McGill University, Montreal Quebec, Canada; ${ }^{2}$ Department of Thoracic Surgery, Memorial Sloan Kettering \\ Cancer Centre, New York, NY, USA \\ Contributions: (I) Conception and design: BJ Park; (II) Administrative support: BJ Park; (III) Provision of study materials or patients: None; (IV) \\ Collection and assembly of data: All authors; (V) Data analysis and interpretation: All authors; (VI) Manuscript writing: All authors; (VII) Final \\ approval of manuscript: All authors. \\ Correspondence to: Jonathan Cools-Lartigue, MD, PhD. Department of Thoracic Surgery, McGill University, Montreal Quebec, Canada. \\ Email: jonathan.cools-lartigue@mcgill.ca.
}

\begin{abstract}
Minimally invasive techniques have rapidly been adopted in the surgical management of thoracic pathologies. These modalities including video-assisted thoracic surgery (VATS) and more recently roboticassisted thoracic surgery (RATS) confer a number of advantages including enhanced recovery and the potential for improved surgical precision. However, the implementation of minimally invasive techniques does result in increased up-front costs associated with the acquisition of necessary equipment. Furthermore, particularly early in the implementation of a minimally invasive approach, increased operative times are incurred. However, these disadvantages may be offset by the purported benefits of a minimally invasive approach, which collectively may reduce hospital length of stay and complication rate. As the costs of care come under greater scrutiny an understanding of both the prevalence of minimally invasive thoracic surgery and its associated drivers of cost is imperative. Herein, the costs associated with minimally invasive thoracic surgical oncologic procedures are reviewed.
\end{abstract}

Keywords: Video-assisted thoracic surgery (VATS); minimally invasive esophagectomy (MIE); esophagectomy; robotic surgery; lung cancer; esophageal cancer

Received: 08 June 2018; Accepted: 21 August 2018; Published: 04 December 2018.

doi: $10.21037 /$ shc.2018.11.05

View this article at: http://dx.doi.org/10.21037/shc.2018.11.05

\section{Introduction}

Lung cancer remains the most common cause of cancer related mortality in the United States with an estimated incidence of 234,030 cases/year and 154,050 deaths/year. Along these lines, 120,000 anatomic lung resections are performed in the United States annually (1). Open thoracotomy remains the most common approach nationwide with an estimated $55 \%$ performed in this manner. The remaining $45 \%$ are predominantly performed by VATS , with adoption increasing most rapidly among thoracic surgeons (2). The minority of pulmonary resections are being performed robotically. However, the former technique is rapidly gaining popularity due to enhanced precision, ergonomics and market factors $(3,4)$. Similarly, minimally invasive techniques have been increasingly employed in the management of esophageal cancer. As the incidence of this disease, particularly esophageal adenocarcinoma, continues to rise, the need for surgical management continues to increase. As esophagectomy, either alone, or as part of a multimodality approach continues to form a mainstay of curative intent management, a clear understanding of its utilization is critical when dealing with this patient population. This procedure, regardless of how it is performed, is technically complex and associated with relatively high rates of morbidity (as high as 50\% in some series) and mortality (5-20\%). The adoption of a minimally invasive approach 
Table 1 Published studies comparing costs incurred following lobectomy

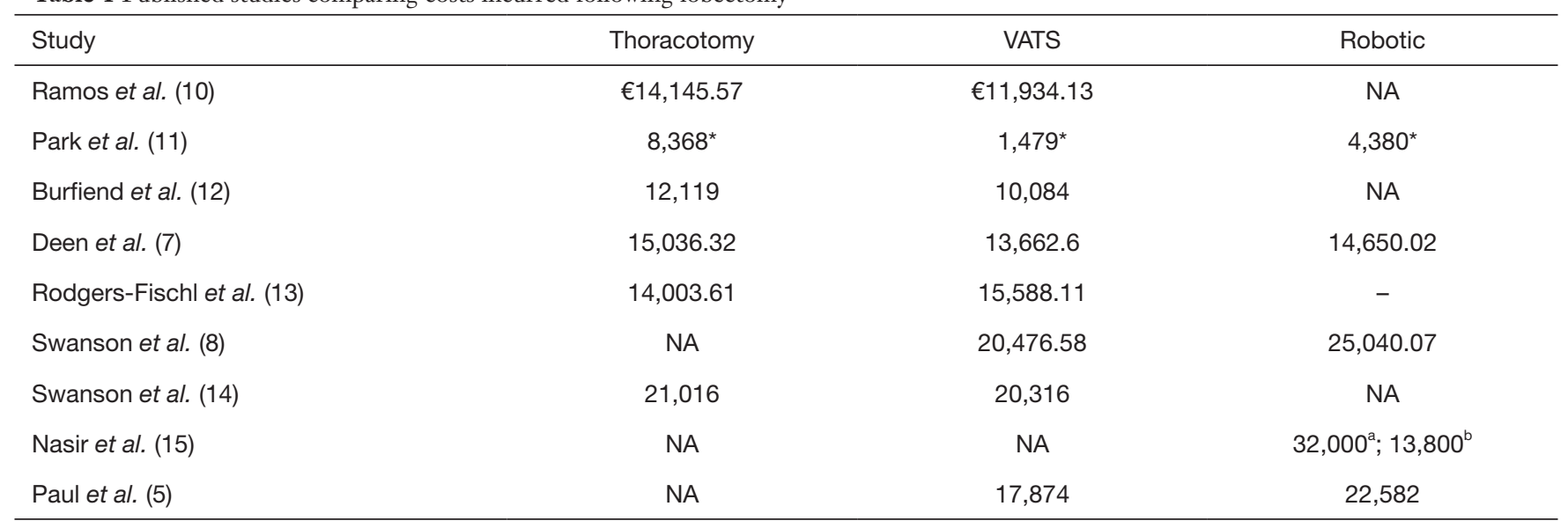

Costs are reported in US dollars per patient unless otherwise specified. *, reflect comparative costs; ${ }^{\text {a }}$, reflects total amount charged by hospital; ${ }^{b}$, reflects operative costs. NA, not applicable; VATS, video-assisted thoracic surgery.

has been done in part with the hopes of improving surgical outcomes in this high-risk population. Of the nearly 4,500 esophagectomies performed in the United States per year, approximately $70 \%$ are performed via the open approach. The remaining $30 \%$ are approached via combined laparoscopic/thoracoscopic approaches with only a small minority being performed robotically. As with lung surgery, a combination of factors has led to increased utilization of minimally invasive techniques, particularly a robotic approach.

Given the rapidly changing technical landscape in the management of patients with thoracic oncologic malignancies, a clear understanding of the value of these approaches is imperative to facilitate optimal patient management. In this context, value refers to the concept of outcomes achieved relative to dollars spent and its assessment is increasingly in focus given the costs associated with the delivery of health care in the modern era. The rapid adoption of cutting edge, albeit expensive techniques mandates an understanding of their relative value as this considers not only the costs associated with the adoption of a new technology, but also the costs related to how a patient is managed throughout their surgical care, from the operating room right through to recovery. Thus, while a new technology may require a significant upfront investment, if it improves patient outcomes, is associated with a reduction in complications or other unforeseen benefits, it may prove to be highly cost effective as a result. Accordingly, the costs associated with the adoption of minimally invasive techniques in pulmonary and esophageal malignancies is reviewed in the present manuscript.

\section{Lung cancer}

As previously stated, open surgery remains the most common method of anatomic lung resection in the United States. Multiple factors contribute to the overall cost of the procedure including initial equipment costs, operative duration, complication rate, and hospital length of stay $(5,6)$. Several contemporary studies have compared the overall cost of VATS lobectomy for lung cancer to open thoracotomy. Overall, VATS lobectomy demonstrates an improved or equivalent cost profile compared to thoracotomy, with the preponderance of current studies demonstrating a cost benefit with respect to the former (7-9). The studies specifically documenting costs are outlined in Table 1.

In the study by Ramos et al., patients undergoing posterolateral thoracotomy or VATS lobectomy were compared retrospectively with respect to patient outcomes and cost. Overall, equipment costs were significantly increased in the VATS group compared to open surgery $(€ 1,800 \pm 560.46$ vs. $€ 901 \pm 328, \mathrm{P}<0.05)(10)$. In addition, operative times were increased in the VATS compared to open groups further adding to up front hospital costs (219 $v s .142 \mathrm{~min}$ total VATS $v s$. open $2,861 \pm 458$ vs. $2,260 \pm 399 \mathrm{~min}$ ). However, these initial costs were offset by a significant reduction in hospital stay leading to a net reduction in overall expenditures (€11,934 vs. €14,145) (10). Additional studies have demonstrated a cost benefit of VATS over open surgery predominantly related to a reduction in hospital stay. In the study by Park et al., an estimated cost saving of 5,098 was attributed directly to a reduction in hospital stay by 2 days compared to open surgery (11). Similarly, 
Burfeind et al. assessed the costs associated with lobectomy in 37 patients subject to posterolateral thoracotomy and 76 patients undergoing VATS. Hospital costs were significantly lower in the VATS group compared to the open group $(\$ 7,144 \pm 2,148$ vs. $\$ 8,251 \pm 2,958, \mathrm{P}=0.0012)$. This benefit was driven by reduced hospital length of stay ( 3 vs. 5 days, $\mathrm{P}=0.0009$ ) as patients were equivalent in terms of overall complication rate (12). Furthermore, patient characteristics were equivalent at baseline thus precluding the possibility of more challenging or complex patents in the open group skewing the cost in favour of a minimally invasive approach. The authors estimated an annual saving of $\$ 100$ million/year across the US following implementation of a similar minimally invasive strategy nationwide (12).

In keeping with these findings, studies in which patients fail to demonstrate an improvement in length of stay fail to demonstrate a significant benefit with respect to cost. In the study by Deen et al., patients undergoing VATS vs. open lobectomy were compared retrospectively with regard to a number of clinical outcomes including length of stay (LOS), overall complication rate and cost. In this study, a small but insignificant improvement in LOS was observed in VATS compared to open lobectomies ( 4.75 vs. 5.47 days $\mathrm{P}=0.77$ ). The overall cost of lobectomy was similar regardless of the approach employed ( $\$ 13,829$ via VATS and $\$ 15,036$ via thoracotomy). Thus, without reduction in overall LOS, no offset to the additional costs of a VATS approach are observed (7). Similarly, Rodgers-Fischl et al. compared 61 patients undergoing VTAS lobectomy to 48 patients undergoing thoracotomy for NSCLC. Overall hospital costs were $\$ 14,003$ vs. $\$ 15,588$ respectively $(\mathrm{P}=0.88)$. The authors noted that in their small retrospective series, there was an insufficient observed reduction in hospital stay (5.5 days VATS $v s$. 5.7 days open) to offset the disposable costs associated with VATS (13).

In the study by Swanson et al., 3,961 patients undergoing lobectomy for documented lung cancer were reviewed. Of these, 2,907 were performed via thoracotomy and 1,054 were performed by VATS (14). Operative times were significantly longer in the VATS group compared to the open group (4.09 vs. 3.75 hours, $\mathrm{P}<0.001$ ). However, VATS was associated with a significant reduction in LOS and need for prolonged hospital stay $>14$ days $(7.83$ vs. 6.15 days, LOS OR $\geq 14$ days $1.53,95 \%$ CI: $1.13-2.09$, respectively). These findings remained significant after adjusting for potential confounders. In keeping with these findings, the open group demonstrated a significantly higher risk of adverse events (OR 1.22, 95\% CI: 1.03-1.44). Overall this translated into a significant reduction in cost in favour of VATS lobectomy. Hospital costs following open lobectomy were $\$ 21,016$ vs. $\$ 20,316$ for VATS. This cost saving was more pronounced when considering only high-volume surgeons (defined as $>16$ surgeries in a 6 -month period, $\$ 18,133$ vs. $\$ 22,050$ in low volume practitioners) $(8,14)$.

When taken together, the results of these studies suggest that when considering the benefit of minimally invasive surgery from the perspective of cost only, the greatest benefit is realized in patients in whom early discharge is possible. It is important to note however, that rapid discharge may be associated with increasing rates of readmission, which themselves would offset the cost benefits associated with early discharge and a minimally invasive approach. This highlights the importance of an appropriately implemented minimally invasive program wherein complications are minimized allowing for patients to derive the maximal benefit with respect to recovery. From the institutional perspective, the improved outcomes translate to reduced costs. This thesis has been studied directly. At the national level minimally invasive approaches are associated with reduced hospital length of stay (16). For example, Rosen et al. queried the national cancer database to identify patients undergoing thoracic surgery for NSCLC in whom hospital LOS and readmission rates were recorded (16). A total of 59,734 patients were identified. Overall, minimally invasive techniques were associated with a reduction in hospital stay (median 5 vs. 6 days). Furthermore, hospitals that employed routine rapid discharge practices were more likely to employ minimally invasive techniques and had lower readmission rates compared to hospitals employing more prolonged discharge practices (16). For example, hospitals demonstrating discharge practices 4 days shorter than the national average exhibited readmission rates of $2.17 \%$ compared to hospitals with mean LOS 1 or more days longer than the national average (4.03-5.43\% readmission rate).

The bulk of cost accrued in hospital related to VATS lobectomy are derived from OR costs (39\%) and inpatient ward costs (26\%) (16). Additional factors associated with incurred costs include urinary tract infections, unplanned transfusion and prolonged air leak $>5$ days. Similarly, factors associated with increased costs in patients undergoing VATS lobectomy include chronic obstructive pulmonary disease (COPD), coronary artery disease (CAD) and $\mathrm{BMI}>35 \mathrm{~kg} / \mathrm{m}^{2}$ (16). Factors associated with readmission included patient age, male sex, pathologic stage, grade and increasing comorbid status. A careful consideration 
of these factors is therefore necessary in order to ensure high quality outcomes in patients undergoing VATS lobectomy while ensuring cost effectiveness. Along these lines, at the national level early discharge practices tend to be overrepresented in academic high volume centres. Poor selection of VATS candidates, or overly zealous discharge practices in themselves are not sufficient to minimize costs as demonstrated by the finding that rapid implementation of a minimally invasive program may be associated with increased complication rates thus offsetting any of the potential benefits from both an economic and patient centred point of view $(17,18)$. For example, Medbery et al. demonstrated increased readmission rates in patients from rural regions who underwent a minimally invasive procedure at a nonacademic institution. This finding highlights the importance of patient selection at multiple levels, starting preoperatively with respect to co-morbid status right through to their socioeconomic situation following discharge as these factors will impact both length of stay and readmission rates regardless the application of a minimally invasive approach (17).

\section{Robotic-assisted thoracoscopic surgery (RATS)}

Contemporary studies have also compared costs associated with the increasing implementation of robotic procedures $(3-5,8,11,15)$. The bulk of costs incurred for robotic cases are due to operating room/equipment costs and ward costs. In the study by Deen et al. the costs for lobectomy were compared amongst VATS, open and robotic modalities. Overall, robotic cases were the most expensive approach, incurring an average additional cost of $\$ 2,100$ compared to VATS cases and $\$ 1,975$ compared to open cases. These differences reached statistical significance. No difference with respect to complication rate or hospital LOS was appreciated between both MIS modalities (32\% vs. 31\% and 4.65 vs. 4.75 days, respectively). However, operative time was significantly longer in robotic cases compared to both open and VATS cases (223 vs. 202 vs. 180, min respectively). Equipment costs were significantly higher in robotic cases compared to open but not VATS cases. Collectively, this data suggests that compared to VATS much of the additional costs are due to increased operative time and associated personnel and equipment costs to a lesser extent overall (7).

The results of the previous study were corroborated by a larger study by Swanson et al. (8). Therein, 15,502 minimally invasive lobectomy and wedge resections were reviewed retrospectively. Compared to VATS procedures RATS were significantly more expensive with an average cost of $\$ 25,040 v s . \$ 20,476(\mathrm{P}<0.001)$ for lobectomies and $\$ 19,592$ vs. $\$ 16,600(\mathrm{P}=0.001)$. Length of stay did not differ between the two groups (6 and 5.23 days RATS vs. 5.83 days VATS for lobectomy and wedge resection respectively $\mathrm{P}=$ not significant). Duration of surgery for patients undergoing lobectomy was longer in the RATS group compared to VATS although this did not reach statistical significance (4.49 vs. 4.23 hours respectively, $\mathrm{P}=0.095)$. In patients undergoing wedge resection, RATS was associated with a significant increase in operative duration compared to VATS (3.3 vs. 2.9 hours respectively, $\mathrm{P}=0.003)$. Collectively RATS was associated with additional incurred cost amounting to $\$ 4,565$ for lobectomy and $\$ 2,992$ for wedge resection compared to VATS. In addition, the authors go on to point out that these costs do not account for the initial robotic acquisition costs which are on the order of 2.5 million dollars, nor do they consider the annual maintenance costs ranging from \$100-180,000.

Despite these findings, appropriate implementation of a robotic surgical program can demonstrate profitability in the current US healthcare environment (15). For example, Nasir et al. reviewed the results of 394 consecutive robotic anatomic resections for NSCLC. Total hospital costs per patient were estimated at $\$ 15,440$. At the time of the study, median Medicare reimbursement per patient was $\$ 18,937$ per patient with a median profit margin of $\$ 3,497$ per patient. It is important to note however, that average LOS was 2 days in the single centre, single surgeon experience outlined. Furthermore, both morbidity and mortality rates were below the rates outlined in the current STS database suggesting that profitability in the current series is highly dependent on excellent surgical outcomes which may not be representative of the national experience.

In fact, not all of the data published thus far replicate the outcomes referenced above. For example, In the study by Paul et al., the outcomes of 2,598 RATS and 37,595 VATS procedures were compared with respect to outcomes including operative time, morbidity, mortality and cost (5). Patients were identified from a national database between 2008-2011. Overall, patients who underwent RATS procedures were more likely to suffer cardiovascular complications other than $\mathrm{MI}$ and iatrogenic intra-operative bleeding complications. Along these lines, patients who underwent RATS were more likely to be subject to nonroutine discharge procedures meaning they were more likely to be discharged to a facility before being sent home. 
Table 2 Published studies comparing costs incurred following esophagectomy

\begin{tabular}{lcc}
\hline Study & Open & MIE \\
\hline Yanasoot et al. (22) & 4,254 & 6,163 \\
Parameswaran et al. (23) & 7,017 & 7,885 \\
Fu et al. (20) & 35,002 & 35,508 \\
Dhamija et al. (24) & 25,935 & 24,440 \\
\hline
\end{tabular}

Costs are reported in US dollars per patient unless otherwise specified. MIE, minimally invasive esophagectomy.

When looking at the data collectively, the distribution of hospitals performing robotic procedures were not uniform. Robotic technology was more frequently applied by smaller $(<1,000$ beds) non-academic institutions in the south. These factors all contributed to an increased cost for robotic surgery compared to VATS $(\$ 22,582$ vs. $\$ 17,874$ respectively, $\mathrm{P}<0.001)$. When considered collectively, the data on robotic surgery suggests that in appropriately structured, high volume centres, excellent outcomes and value can be achieved. However, this does not represent the implementation of existing robotic thoracic surgical programs nationwide.

\section{Esophageal cancer}

Minimally invasive techniques have similarly been adopted with increasing frequency in the management of esophageal cancer. As with their application in lung resection, concerns regarding the safety and costs of minimally invasive esophagectomy (MIE) compared to open surgery have been raised. With respect to the former, a number of prospective and randomized studies have demonstrated that minimally invasive approaches are associated with shorter LOS, reduced blood loss and reduced incidence of pulmonary complications (19). However, a relative paucity of data comparing the costs of traditional techniques compared to MIE exists in the literature today.

Contemporary data comparing MIE and open esophagectomy have demonstrated comparable safety and oncologic outcomes between the two approaches with a consistent trend towards decreased LOS following MIE (19-22). However, greater variability exists with regards to quantifying value associated with the two techniques, with findings ranging from increased to decreased overall costs. The few studies specifically outlining costs are listed in Table 2. For example, in the study by Parameswaran et al., operative outcomes were compared between cohorts of patients with esophageal cancer undergoing esophagectomy via open and combined thoracoscopic/laparoscopic minimally invasive approaches. Increased costs were incurred among patients in the MIE group (\$7,017 open vs. $\$ 7,885 \mathrm{MIE}$ ), although overall they were quite similar (23). Furthermore, the bulk of the incurred costs were attributed to increased operative costs of which operative time was the most significant contributor (23). However, the authors noted that operative time decreased significantly after 30 cases as surgeon experience increased. Similarly, the study by Yanasoot et al. demonstrated increased costs associated with hybrid and completely minimally invasive approached in patients undergoing esophagectomy for malignancy (22). As in the previous study, this was associated with markedly increased operative times in the minimally invasive group. The approach however, was not associated with increased postoperative complications nor increased overall length of stay, suggesting that the increased costs were almost exclusively due to operative costs (1). Conversely, in the study by Lee $e t a l$., an overall cost advantage was observed in patients undergoing MIE (25). Using a decision analysis model, the authors were able to estimate the total costs incurred in patients undergoing open esophagectomy or MIE. While operative costs related to equipment and operative time were increased, an overall cost benefit was observed. This was related to reduced LOS and reduced postoperative complications. Thus overall, MIE was found to be a more cost effective procedure when compared to open esophagectomy within a framework that resulted in fewer complications and the ability to reduce stay. Even in the context of reduced LOS, MIE has not consistently demonstrated a cost advantage over an open approach. In the study by Dhamija et al. a series of 160 esophagectomy patients were reviewed with respect to operative outcomes and costs (24). The authors demonstrated that while median LOS were significant shorter in the MIE compared to open group (11 vs. 19 days, $\mathrm{P}=0.006$ ) no cost advantage could be demonstrated $(\$ 25,935$ MIE vs. $\$ 24,440$ open $\mathrm{P}=\mathrm{NS})$. However, while no difference in mortality was noted, the authors do not specifically address overall morbidity in their series. Thus LOS is not the sole driver of reduced costs when applying MIE (24).

The discrepancy in costs observed in the previously mentioned studies suggests that the financial implications related to the adoption of MIE are multifactorial. Esophagectomy is a highly complex procedure and surgical outcomes are often related to more than isolated 
technical complications. Furthermore, the management of these complications, such as anastomotic leak, are often prolonged and require repeat interventions, which themselves are likely to incur significant costs. In keeping with these observations, additional studies have supported the notion that overall hospital costs are related complication rates as opposed to the operative technique employed in patients undergoing esophagectomy. For example, in the study by Fu et al., overall hospital costs between MIE and open esophagectomy were similar despite increased operative time and operative costs in MIE. Length of stay was identical regardless of surgical approach and was therefore not associated with cost savings in the MIE group. Conversely, costs incurred following surgery related to hospital services such as physiotherapy were significantly reduced, accounting for the overall similarity in expenditures. Thus, on average, esophagectomy was associated with an expenditure of $\$ 31,375$ per patient, ranging between $\$ 27,800$ for THE to 35,500 for MIE. This being said, patients who required re-intubation incurred an additional cost of $\$ 20,000$ while those who experienced an anastomotic leak incurred an average additional cost of $\$ 14,000$ (20). When taken together, the results of contemporary studies suggest that MIE can be adopted in a cost-effective manner, provided it is employed in a setting that leads to improved outcomes and reduced complications. In this context, no significant cost disadvantage is observed when adopting a minimally invasive surgical approach to esophageal cancer $(19,20,25)$.

\section{Robotic-assisted minimally invasive esophagectomy (RAMIE)}

With respect to the introduction of robotic techniques, this procedure has demonstrated comparable results to both open and MIE from the standpoint of patient morbidity and mortality $(11,26)$. Furthermore, it is associated with a significantly shorter learning curve potentially allowing for more widespread adoption of minimally invasive techniques in this field $(27,28)$. To date however, costs remain elevated compared to both open and combined thoracoscopic/laparoscopic techniques. The study by Suda et al. suggests an average cost increase of approximately $\$ 1,500$ per case independent of the tremendous upfront costs associated with system purchase ( $\$ 2$ million) and maintenance (\$150,000 annually) (29). Similarly, operative times in contemporary series have been significantly longer during RAMIE compared to either open or combined laparoscopic/thoracoscopic approaches, with early experiences listing times as high as 11 hours (30). However, significant reductions can be expected with increasing surgeon experience as demonstrated by Cerfolio et al., wherein operative times were reduced to $>350$ minutes following 15-22 cases when employing a 2-layered hand sewn intrathoracic anastomosis (31). As technology becomes more widely available and surgeon experience grows, it is likely that costs will continue to decline, further expanding application of RAMIE.

\section{Conclusions}

Collectively, the data published in both the lung and esophageal cancer literature suggest that adoption of a minimally invasive technique in thoracic surgery has the ability to improve patient outcomes and add value to patient care. This is predicated on implementation that permits enhanced recovery and early discharge without incurring additional complications. With respect to robotic procedures, upfront costs are significant and currently limit the application of this technique based on value alone. Furthermore, efficient and safe application of a robotic thoracic program requires significant multidisciplinary expertise in order to achieve favorable outcomes with acceptable cost at this time. This being said, randomized studies comparing robotic to open approaches are underway, and based on the literature thus far, are likely to demonstrate similar benefits over open techniques while expanding their adoption. Thus, while a myriad of options exists in the management of thoracic malignancies, a reasonable value-based approach requires that regardless of the technique employed, complications are minimized and patient management and recovery are prioritized.

\section{Acknowledgments}

Funding: None.

\section{Footnote}

Provenance and Peer Review: This article was commissioned by the Guest Editors (Lorenzo Spaggiari and Domenico Galetta) for the series "Minimally Invasive Thoracic Oncological Surgery" published in Shanghai Chest. The article has undergone external peer review.

Conflicts of Interest: Both authors have completed the 
ICMJE uniform disclosure form (available at http://dx.doi. org/10.21037/shc.2018.11.05). The series "Minimally Invasive Thoracic Oncological Surgery" was commissioned by the editorial office without any funding or sponsorship. The authors have no other conflicts of interest to declare.

Ethical Statement: The authors are accountable for all aspects of the work in ensuring that questions related to the accuracy or integrity of any part of the work are appropriately investigated and resolved.

Open Access Statement: This is an Open Access article distributed in accordance with the Creative Commons Attribution-NonCommercial-NoDerivs 4.0 International License (CC BY-NC-ND 4.0), which permits the noncommercial replication and distribution of the article with the strict proviso that no changes or edits are made and the original work is properly cited (including links to both the formal publication through the relevant DOI and the license). See: https://creativecommons.org/licenses/by-nc-nd/4.0/.

\section{References}

1. Cronin KA, Lake AJ, Scott S, et al. Annual Report to the Nation on the Status of Cancer, part I: National cancer statistics. Cancer 2018;124:2785-800.

2. Blasberg JD, Seder CW, Leverson G, et al. Video-Assisted Thoracoscopic Lobectomy for Lung Cancer: Current Practice Patterns and Predictors of Adoption. Ann Thorac Surg 2016;102:1854-62.

3. Mungo B, Hooker CM, Ho JS, et al. Robotic Vs. Thoracoscopic Resection for Lung Cancer: Early Results of a New Robotic Program. J Laparoendosc Adv Surg Tech A 2016;26:243-8.

4. Oh DS, Reddy RM, Gorrepati ML, et al. Robotic-Assisted, Video-Assisted Thoracoscopic and Open Lobectomy: Propensity-Matched Analysis of Recent Premier Data. Ann Thorac Surg 2017;104:1733-40.

5. Paul S, Jalbert J, Isaacs AJ, et al. Comparative effectiveness of robotic-assisted vs. thoracoscopic lobectomy. Chest 2014;146:1505-12.

6. Klapper J, D'Amico TA. VATS vs. open surgery for lung cancer resection: moving toward a minimally invasive approach. J Natl Compr Canc Netw 2015;13:162-4.

7. Deen SA, Wilson JL, Wilshire CL, et al. Defining the cost of care for lobectomy and segmentectomy: a comparison of open, video-assisted thoracoscopic, and robotic approaches. Ann Thorac Surg 2014;97:1000-7.
8. Swanson SJ, Miller DL, McKenna RJ Jr, et al. Comparing robot-assisted thoracic surgical lobectomy with conventional video-assisted thoracic surgical lobectomy and wedge resection: results from a multihospital database (Premier). J Thorac Cardiovasc Surg 2014;147:929-37.

9. Kuritzky AM, Aswad BI, Jones RN, et al. Lobectomy by Video-Assisted Thoracic Surgery vs. Muscle-Sparing Thoracotomy for Stage I Lung Cancer: A Critical Evaluation of Short- and Long-Term Outcomes. J Am Coll Surg 2015;220:1044-53.

10. Ramos R, Masuet C, Gossot D. Lobectomy for early-stage lung carcinoma: a cost analysis of full thoracoscopy vs. posterolateral thoracotomy. Surg Endosc 2012;26:431-7.

11. Park BJ, Flores RM. Cost comparison of robotic, videoassisted thoracic surgery and thoracotomy approaches to pulmonary lobectomy. Thorac Surg Clin 2008;18:297300 , vii.

12. Burfeind WR Jr, Jaik NP, Villamizar N, et al. A costminimisation analysis of lobectomy: thoracoscopic vs. posterolateral thoracotomy. Eur J Cardiothorac Surg 2010;37:827-32.

13. Rodgers-Fischl PM, Martin JT, Saha SP. Video-Assisted Thoracoscopic vs. Open Lobectomy: Costs and Outcomes. South Med J 2017;110:229-33.

14. Swanson SJ, Meyers BF, Gunnarsson CL, et al. Videoassisted thoracoscopic lobectomy is less costly and morbid than open lobectomy: a retrospective multiinstitutional database analysis. Ann Thorac Surg 2012;93:1027-32.

15. Nasir BS, Bryant AS, Minnich DJ, et al. Performing robotic lobectomy and segmentectomy: cost, profitability, and outcomes. Ann Thorac Surg 2014;98:203-8; discussion 208-9.

16. Rosen JE, Salazar MC, Dharmarajan K, et al. Length of Stay From the Hospital Perspective: Practice of Early Discharge Is Not Associated With Increased Readmission Risk After Lung Cancer Surgery. Ann Surg 2017;266:383-8.

17. Medbery RL, Perez SD, Force SD, et al. Video-assisted thoracic surgery lobectomy cost variability: implications for a bundled payment era. Ann Thorac Surg 2014;97:168692; discussion 1692-3.

18. Medbery RL, Gillespie TW, Liu Y, et al. Socioeconomic Factors Are Associated With Readmission After Lobectomy for Early Stage Lung Cancer. Ann Thorac Surg 2016;102:1660-7.

19. Straatman J, van der Wielen N, Cuesta MA, et al. Minimally Invasive Vs. Open Esophageal Resection: Three-year Follow-up of the Previously Reported Randomized Controlled Trial: the TIME Trial. Ann Surg 
2017;266:232-6.

20. Fu SJ, Ho VP, Ginsberg J, et al. Complications, Not Minimally Invasive Surgical Technique, Are Associated with Increased Cost after Esophagectomy. Minim Invasive Surg 2016;2016:7690632.

21. Rodham P, Batty JA, McElnay PJ, et al. Does minimally invasive oesophagectomy provide a benefit in hospital length of stay when compared with open oesophagectomy? Interact Cardiovasc Thorac Surg 2016;22:360-7.

22. Yanasoot A, Yolsuriyanwong K, Ruangsin S, et al. Costs and benefits of different methods of esophagectomy for esophageal cancer. Asian Cardiovasc Thorac Ann 2017;25:513-7.

23. Parameswaran R, Veeramootoo D, Krishnadas R, et al. Comparative experience of open and minimally invasive esophagogastric resection. World J Surg 2009;33:1868-75.

24. Dhamija A, Dhamija A, Hancock J, et al. Minimally invasive oesophagectomy more expensive than open despite shorter length of stay. Eur J Cardiothorac Surg 2014;45:904-9.

25. Lee L, Sudarshan M, Li C, et al. Cost-effectiveness of

doi: $10.21037 /$ shc.2018.11.05

Cite this article as: Cools-Lartigue J, Park BJ. Economic assessment in minimally invasive thoracic oncological surgeryUSA experience. Shanghai Chest 2018;2:89. minimally invasive vs. open esophagectomy for esophageal cancer. Ann Surg Oncol 2013;20:3732-9.

26. Yerokun BA, Sun Z, Yang CJ, et al. Minimally Invasive Vs. Open Esophagectomy for Esophageal Cancer: A Population-Based Analysis. Ann Thorac Surg 2016;102:416-23.

27. Park S, Hyun K, Lee HJ, et al. A study of the learning curve for robotic oesophagectomy for oesophageal cancer. Eur J Cardiothorac Surg 2018;53:862-70.

28. Zhang H, Chen L, Wang Z, et al. The Learning Curve for Robotic McKeown Esophagectomy in Patients With Esophageal Cancer. Ann Thorac Surg 2018;105:1024-30.

29. Suda K, Nakauchi M, Inaba K, et al. Robotic surgery for upper gastrointestinal cancer: Current status and future perspectives. Dig Endosc 2016;28:701-13.

30. Lehenbauer D, Kernstine KH. Robotic esophagectomy: modified McKeown approach. Thorac Surg Clin 2014;24:203-9, vii.

31. Cerfolio RJ, Bryant AS, Hawn MT. Technical aspects and early results of robotic esophagectomy with chest anastomosis. J Thorac Cardiovasc Surg 2013;145:90-6. 